Journal of Thermal Engineering, Vol. 6, No. 2, Special Issue 11, pp. 24-40, March, 2020

Yildiz Technical University Press, Istanbul, Turkey

\title{
DIFFERENTIAL TRANSFORMATION METHOD FOR ANALYSIS OF NONLINEAR FLOW AND MASS TRANSFER THROUGH A CHANNEL FILLED WITH A POROUS MEDIUM
}

\author{
M. Hatami ${ }^{1}{ }^{\star}$, S. Mosayebidorcheh², M. Vatani ${ }^{3}$, T. Mosayebidorcheh², D.D. Ganji ${ }^{3}$
}

\begin{abstract}
In this paper, mass transfer and chemical reaction effects on laminar viscous flow through a porous channel with moving or stationary walls are studied. The governing partial differential equations of the physical problem are transformed into a set of coupled nonlinear ordinary differential equations using similarity transformation. The coupled nonlinear ordinary differential equations are solved using differential transform method (DTM). The results obtained through the approximate analytical method are compared with the results of numerical method and high accuracy of the present approximate analytical solution is observed. The valuable achievement of the present study is imbedding a precise and efficient analytical method for the flow of viscous fluid in a porous channel with a chemical reaction. Also, the effects of some pertinent parameters such as Reynolds number, Darcy number, Schmidt number and suction/injection parameter on velocity components, heat transfer, concentration, and Sherwood distribution are presented in this work.
\end{abstract}

Keywords: Heat Transfer, Chemical Reaction, Porous Channel; Boundary Value Problem; Differential Transformation Method (DTM)

\section{INTRODUCTION}

Most of scientific problems in fluid mechanics and dynamics are innately nonlinear. All of these problems are displayed by partial or ordinary differential equations. The problem of flow and mass transfer in a porous media with a chemical reaction is an example of system of coupled nonlinear differential equations which can be solved by analytical methods such as DTM.

The flow in the porous media with a chemical reaction frequently occurs in many physical problems and engineering applications such as filtration processes, combustion systems, geothermal energy extraction systems, oil and gas production and chemical engineering [1-4]. In review of the importance of this problem, the flow characteristics have been investigated by numerous authors. Beg and Makinde [5] analyzed the laminar flow of an upper-convected Maxwell (UCM) viscoelastic fluid with species diffusion in a Darcian high-permeability porous channel using 6th order Rung-Kutta method. Hayat and Abbas [6] studied the flow of UCM fluid in a porous channel with chemical reaction in which the effects of Deborah number, Reynolds number, Schmidt number and chemical reaction parameter on the velocity and concentration distribution are examined. Rundora and Makinde [7] investigated the thermal effects of suction or injection on an unsteady reactive variable viscosity third grade fluid in a porous media subject to convective boundary condition using finite difference method. The obtained results reveal that the velocity and temperature profile are both decreased by increasing injection/suction Reynolds number. Chinyoka and Makinde [8] presented the transient solution of the flow of a reactive variable viscosity fluid in a circular pipe with a porous wall using semi-implicit finite difference method. Srinivas and Muthuraj [9] studied the influence of MHD mixed convection flow through a vertical asymmetric channel with chemical reaction. The momentum, energy and concentration equations of the problem have been linearized using long-wavelength approximation. In addition, other works [10-15] also investigated the flow and heat transfer in the porous medium for different conditions.

This paper was recommended for publication in revised form by Regional Editor Omid Mahian

${ }^{1}$ Department of Mechanical Engineering, Esfarayen University of Technology, Esfarayen, North Khorasan, Iran

2 Young Researchers and Elite Club, Najafabad Branch, Islamic Azad University, Najafabad,Iran

${ }^{3}$ Department of Mechanical Engineering, Babol University of Technology, Babol, Iran

${ }^{*}$ E-mail address: m.hatami2010@gmail.com

Orcid id: https://orcid.org/0000-0001-5657-6445

Manuscript Received 28 May 2017, Accepted 08 August 2017 

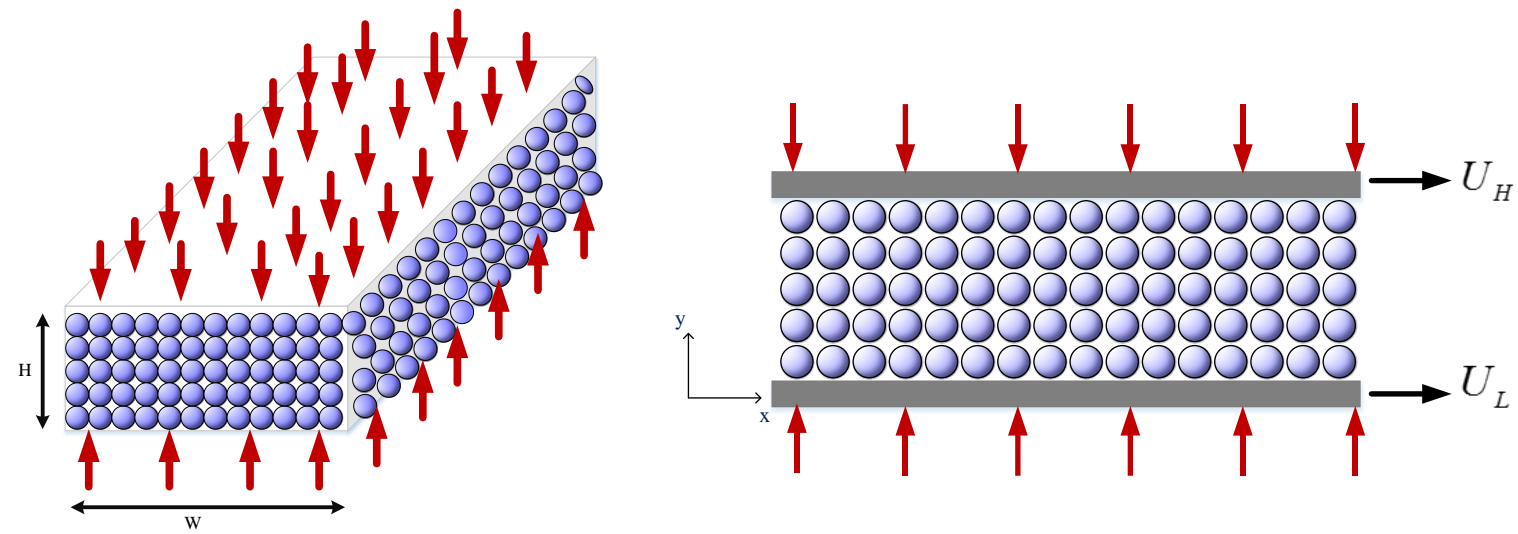

Figure 1. The physical model of flow geometry

The effects of internal heat generation and chemical reaction on free convective of polar fluid in a porous medium is studied by Patil and Kulkarni [16]. The results show that the presence of chemical reaction and internal heat source affect the flow field considerably. Reddy et al. [17] solved the heat and mass transfer equations of asymmetric laminar flow in a porous channel with expanding or contracting walls having different permeability in the presence of chemical reaction. Matin and Pop [18] investigated the fully developed flow of nanofluid through a porous channel with the constant heat flux wall in the presence of the chemical reaction. A Brinkman model and the clear fluid compatible model are applied to derive the governing equations. The heat and mass transfer flow of chemically reactive dusty viscoelastic fluid in a porous channel with convective boundary condition was investigated by Sivaraj and Kumar [19]. They deduced that the velocity and temperature profiles decrease by increasing the radiation parameter and the velocity and concentration distribution also decrease by increasing chemical reaction parameter. Srinivas et al. [20] examined the effects of mass transfer and chemical reaction on laminar flow in a porous channel subject to different boundary condition at the walls. Mahdy [21] studied coupled heat and mass transfer problem on double-diffusive convection from a vertical truncated in porous medium in the presence of chemical reaction with variable viscosity and also the effect of heat generation or absorption on the problem was examined.

Most of the physical problems are nonlinear and do not have an exact analytical solution. So, numerical and approximate methods are used by researchers to solve such equations. The numerical methods are often costly and time consuming to get a complete form of results, because it gives the solution at the discrete points. Furthermore, in the numerical solution the stability and convergence should be considered to avoid divergence or inappropriate results. Approximate techniques like decomposition method (DM), Homotopy Analysis Method (HAM), Homotopy Perturbation Method (HPM), Variational Iteration Method (VIM) are good substitutes for numerical methods. During the recent years, some of the nonlinear engineering problems have been solved using some of these methods, such as HAM [22-25], HPM [26-28], VIM [29-31] and DM [32-34]. In most of the researches, some modifications introduced to overcome the nonlinearity.

Differential transform method (DTM) is also one of the other approximate methods to solve differential equations. This method was introduced by Zhou [35] to solve initial value problems in analysis of the electrical circuits. After that, DTM is applied on differential algebraic equations [36, 37], partial differential equations[38-43], integral equations [44-46], ordinary differential equations [47-50] and fractional differential equations [51-54]. The method is an iterative technique to find the Taylor series solution of the problem. In this method, there is no need to the high calculation cost to determine the coefficients of Taylor series. The main objective of the present work is to analytically study the effects of heat transfer and chemical reaction on viscous laminar flow in a porous channel using differential transformation method. The results obtained in this research in comparison with numerical solution show that the method is efficient and accurate. 
Journal of Thermal Engineering, Research Article, Vol. 6, No. 2, Special Issue 11, pp. 24-40, March, 2020

\section{PROBLEM DESCRIPTION}

Consider steady, laminar, two-dimensional flow of a viscous incompressible fluid through a porous medium in the presence of chemical reaction. The flow regime studying is shown in Fig. 1 including a porous channel with different boundary conditions in a coordinate system. The axis is taken along the direction of the flow and the y is normal to it. By choosing polar coordinates, the governing equations are:

$$
\begin{gathered}
\frac{\partial u}{\partial x}+\frac{\partial v}{\partial y}=0 \\
\frac{\rho}{\varepsilon}\left(u \frac{\partial u}{\partial x}+v \frac{\partial u}{\partial y}\right)=-\frac{\partial p}{\partial x}+\frac{\mu}{\varepsilon}\left(\frac{\partial^{2} u}{\partial x^{2}}+\frac{\partial^{2} u}{\partial y^{2}}\right)-\frac{\mu}{K} u \\
\frac{\rho}{\varepsilon}\left(u \frac{\partial v}{\partial x}+v \frac{\partial v}{\partial y}\right)=-\frac{\partial p}{\partial y}+\frac{\mu}{\varepsilon}\left(\frac{\partial^{2} v}{\partial x^{2}}+\frac{\partial^{2} v}{\partial y^{2}}\right)-\frac{\mu}{K} v
\end{gathered}
$$

Where $u$ and $v$ are the velocity components of $x$ and $y$ direction, $\rho$ is the density, $\mu$ is the fluid viscosity, $\varepsilon$ is the porosity of the porous medium and $K$ is the permeability of the porous media. The related boundary conditions for the flow are:

$$
\begin{gathered}
u=u_{L} \frac{x}{H}, v=v_{L} \text { at } y=0 \\
u=u_{H} \frac{x}{H}, v=v_{H} \text { at } y=H
\end{gathered}
$$

Introducing the following transformations to facilitate the solution:

$$
X=\frac{x}{H}, Y=\frac{y}{H}, P=\frac{p}{\rho v_{L}^{2}}, U=\frac{u}{v_{L}}, V=\frac{v}{v_{L}}
$$

where $v_{H}$ is the normal velocity at the upper wall and $v_{L}$ denotes the normal velocity at the lower wall. An injection occurs at both walls where $v_{L}>0$ and $v_{H}<0$, and pure suction occurs for $v_{L}<0$ and $v_{H}>0$. After substituting the above transformations, the governing equations are reduced to:

$$
\begin{gathered}
\frac{\partial U}{\partial X}+\frac{\partial V}{\partial Y}=0 \\
\frac{1}{\varepsilon}\left(U \frac{\partial U}{\partial X}+V \frac{\partial U}{\partial Y}\right)=-\frac{\partial P}{\partial X}+\frac{1}{\varepsilon \operatorname{Re}}\left(\frac{\partial^{2} U}{\partial Y^{2}}+\frac{\partial^{2} U}{\partial X^{2}}\right)-\frac{U}{\operatorname{Re} \cdot D a} \\
\frac{1}{\varepsilon}\left(U \frac{\partial V}{\partial X}+V \frac{\partial V}{\partial Y}\right)=-\frac{\partial P}{\partial Y}+\frac{1}{\varepsilon \operatorname{Re}}\left(\frac{\partial^{2} V}{\partial X^{2}}+\frac{\partial^{2} V}{\partial Y^{2}}\right)-\frac{V}{\operatorname{Re} \cdot D a}
\end{gathered}
$$


Journal of Thermal Engineering, Research Article, Vol. 6, No. 2, Special Issue 11, pp. 24-40, March, 2020

where $\operatorname{Re}=\frac{H V}{v}$ is the Reynolds number and $D a=\frac{K}{H^{2}}$ Denotes the Darcy number. Now the relevant boundary conditions are:

$$
U(x, 0)=\lambda X, U(X, 1)=\gamma X, V(X, 0)=\alpha, V(X, 1)=\beta
$$

where $\alpha$ is the injection/suction parameter for the lower wall and $\beta=\frac{v_{U}}{v_{L}}$ denotes the injection/suction parameter for the upper wall. $\lambda=\frac{u_{L}}{v_{L}}$ is the axial velocity coefficient for the lower wall and $\gamma=\frac{u_{H}}{v_{L}}$ is for the upper wall. By embedding a suitable change of variables according to the physics of the problem [55], the momentum equation is reduced to:

$$
\left\{\begin{array}{l}
\frac{\partial P}{\partial X}=X\left\{\frac{1}{\operatorname{Re} . \varepsilon} F^{\prime \prime \prime}+\frac{1}{\varepsilon}\left(F^{\prime \prime} F-F^{\prime 2}\right)-\frac{1}{\operatorname{Re} \cdot D a} F^{\prime}\right\} \\
\frac{\partial P}{\partial Y}=\left\{-\frac{1}{\operatorname{Re} . \varepsilon} F^{\prime \prime}-\frac{1}{\varepsilon} F F^{\prime}+\frac{1}{\operatorname{Re} \cdot D a} F\right\}
\end{array}\right.
$$

Eliminating the pressure from the Eq. (10) gives:

$$
F^{(4)}+\operatorname{Re}\left(F^{\prime \prime \prime} F-F^{\prime \prime} F^{\prime}\right)-\frac{\varepsilon}{D a} F^{\prime \prime}=0
$$

And then, the boundary condition for the nonlinear ODE can be expressed as follows:

$$
F^{\prime}(0)=\lambda, F^{\prime}(1)=\gamma, F(0)=-\alpha, F(1)=-\beta
$$

Also, the governing mass-spices equation for this problem is expressed as follows:

$$
u \frac{\partial C}{\partial x}+v \frac{\partial C}{\partial y}=D \frac{\partial^{2} C}{\partial y^{2}}-k_{1} C
$$

where $D$ is the species diffusivity, $C$ is the concentration field and $k_{1}$ is the reaction rate constant of a homogeneous first-order chemical reaction. The appropriate boundary conditions of the problem are:

$$
\begin{gathered}
C=C_{L} \text { at } y=0 \\
C=C_{H} \text { at } y=H
\end{gathered}
$$

Defining the concentration of the fluid in the channel $\left(C=C_{L}+A\left(\frac{x}{H}\right)^{m} G(y)\right)$ and non-dimensional transformation for the concentration field ( $G=\frac{C-C_{H}}{C_{L}-C_{H}}$ ), Eq. (13) becomes:

$$
G^{\prime \prime}-\operatorname{Re} m S c F^{\prime} G+\operatorname{Re} S c F G^{\prime}-g S c G-K_{1} S c=0
$$


Journal of Thermal Engineering, Research Article, Vol. 6, No. 2, Special Issue 11, pp. 24-40, March, 2020

and the associated boundary conditions are:

$$
G(0)=1, G(1)=0
$$

Here the Schmidt number ( $S c$ ), the chemical reaction parameter $(g)$ and $K_{1}$ are denoted by:

$$
S c=\frac{v}{D}, g=\frac{k_{1} H^{2}}{v}, K_{1}=\frac{k_{1} C_{0} H^{2}}{v\left(C_{L}-C_{H}\right)}
$$

\section{THE BASIC PRINCIPLE OF DIFFERENTIAL TRANSFORMATION METHOD}

The differential transform is defined as follows:

$$
X(k)=\frac{1}{k !}\left[\frac{d^{k} x(t)}{d t^{k}}\right]_{t=t_{0}} .
$$

Where $\mathrm{x}(\mathrm{t})$ is an arbitrary function, and $\mathrm{X}(\mathrm{k})$ is the transformed function. The inverse transformation is as follows

$$
x(t)=\sum_{k=0}^{\infty} X(k)\left(t-t_{0}\right)^{k} .
$$

Substituting Eq. 0 into the Eq. (1), we have

$$
x(t)=\sum_{k=0}^{\infty} \frac{\left(t-t_{0}\right)^{k}}{k !}\left[\frac{d^{k} x(t)}{d t^{k}}\right]_{t=t_{0}} .
$$

The function $\mathrm{x}(\mathrm{t})$ is usually considered as a series with limited terms and Eq. (1), can be rewritten as:

$$
x(t) \approx \sum_{k=0}^{q} X(k)\left(t-t_{0}\right)^{k} .
$$

Where, q represents the number of Taylor series' components. Usually, through elevating this value, we can increase the accuracy of the solution. Some of the properties of DTM shown in Table 1. These properties are extracted from Eqs. 0 and (1).

\section{APPLICATION OF DTM TO THE FLOW PROBLEM}

In this section, we try to solve the Eqs. 0 and 0 using DTM. The solution consists of two stages, first through mathematical relations and applying DTM properties, the Taylor series of solution is found. After that, the boundary conditions applied on solution to obtain the unknown parameters.

Table 1. The properties of the DTM.

\begin{tabular}{|c|c|}
\hline Original function & DTM \\
\hline$f(t)=g(t) \pm s(t)$ & $F(k)=G(k) \pm S(k)$ \\
\hline$f(t)=c g(t)$ & $F(k)=\frac{(k+n) !}{k !} G(k+n)$ \\
\hline$f(t)=\frac{d^{n} g(t)}{d t^{n}}$ & $F(k)=\sum_{r=0}^{k} G(r) S(k-r)$ \\
\hline$f(t)=g(t) s(t)$ & \\
\hline
\end{tabular}


Journal of Thermal Engineering, Research Article, Vol. 6, No. 2, Special Issue 11, pp. 24-40, March, 2020

The solution of the problem are considered as the Taylor series at $y=0$ in the following form

$$
\begin{array}{lll}
F(y)=\sum_{k=0}^{q} \bar{F}(k) y^{k}, & & 0 \leq y \leq 1 \\
G(y)=\sum_{k=0}^{q} \bar{G}(k) y^{k}, & 0 \leq y \leq 1
\end{array}
$$

To solve problem using DTM, the boundary conditions in Eqs. 0 and 0 should be transformed to initial conditions at $y=0$. So, we have:

$$
\begin{aligned}
& F(0)=-\alpha, \quad F^{\prime}(0)=\lambda, \quad F^{\prime \prime}(0)=a_{1}, \quad F^{\prime \prime \prime}(0)=a_{2}, \\
& G(0)=1, \quad G^{\prime}(0)=a_{3} .
\end{aligned}
$$

where $a_{1}$ to $a_{3}$ are the unknown parameters. By applying the DTM on Eqs. 0 and 0 at $y=0$, the following recursive relations obtained for calculating the series solutions' coefficients

$$
\begin{gathered}
\bar{F}(k+4)=\frac{-1}{(k+1)(k+2)(k+3)(k+4)}\left\{\operatorname{Re} \sum_{r=0}^{k}(r+1)(r+2)(r+3) \bar{F}(r+3) \bar{F}(k-r)\right. \\
\left.-\operatorname{Re} \sum_{r=0}^{k}(r+1)(r+2)(k-r+1) \bar{F}(r+2) \bar{F}(k-r+1)-\frac{\varepsilon}{D a}(k+1)(k+2) \bar{F}(k+2)\right\} \\
\bar{G}(k+2)=\frac{-1}{(k+1)(k+2)}\left\{-\operatorname{Re} m S c \sum_{r=0}^{k}(r+1) \bar{F}(r+1) \bar{G}(k-r)\right. \\
\left.+\operatorname{Re} S c \sum_{r=0}^{k}(r+1) \bar{G}(r+1) \bar{F}(k-r)-g S c \bar{G}(k)-k_{1} S c \delta(k)\right\}
\end{gathered}
$$

the differential transform of the conditions in Eq. 0 is:

$$
\begin{aligned}
& \bar{F}(0)=-\alpha, \quad \bar{F}(1)=\lambda, \quad \bar{F}(2)=\frac{a_{1}}{2}, \quad \bar{F}(3)=\frac{a_{2}}{6}, \\
& \bar{G}(0)=1, \quad \bar{G}(1)=a_{3} .
\end{aligned}
$$

By substituting Eq. 0 in the Eqs. 0 and 0, the coefficients of Taylor series solution can be obtained as a function of the unknown parameters $\left(a_{1}\right.$ to $\left.a_{3}\right)$. Now, we should obtain the unknown parameters from the boundary conditions in Eq.0. Regarding Eq. 0, we apply the boundary conditions at $y=1$.

$$
\begin{aligned}
& F\left(a_{1}, a_{2}, a_{3}, 1\right)=\sum_{k=0}^{q} \bar{F}(k)=-\beta \\
& F^{\prime}\left(a_{1}, a_{2}, a_{3}, 1\right)=\sum_{k=1}^{q} k \bar{F}(k)=\gamma \\
& G\left(a_{1}, a_{2}, a_{3}, 1\right)=\sum_{k=0}^{q} \bar{G}(k)=0
\end{aligned}
$$


Journal of Thermal Engineering, Research Article, Vol. 6, No. 2, Special Issue 11, pp. 24-40, March, 2020

By solving the Eq. 0 , the values of $a_{1}$ to $a_{3}$ can be computed and the Taylor series solution of problem will obtain. The values of the coefficients $F(h), G(h)$ can be obtained in the following equations for $\lambda=0, \gamma=0, \alpha=-1, \beta=0, \operatorname{Re}=1, D a=0.5, \varepsilon=0.1, m=1, S c=0.65, g=1, k_{1}=0.1$.

$$
\begin{aligned}
& F(4)=-0.0416 a_{2}+0.0083 a_{1}, \quad F(5)=0.01 a_{2}-0.0016 a_{1}+0.0083 a_{1}^{2} \\
& F(6)=0.0027 a_{1} a_{2}-0.0019 a_{2}+0.0003 a_{1}-0.0013 a_{1}^{2}, \ldots \\
& G(2)=-0.325 a_{3}+0.3575, \quad G(3)=0.1083 a_{1}+0.1787 a_{3}-0.07745 \\
& G(4)=0.0270 a_{1} a_{3}+0.0270 a_{2}-0.0176 a_{1}-0.0466 a_{3}+0.03195, \ldots
\end{aligned}
$$

finally, regarding to the boundary conditions the unknown values and the approximate solution can be obtained:

$$
\begin{gathered}
F(y)=-0.7343+0.5312 y-0.3901 y^{2}+0.1868 y^{3}-0.0500 y^{4}+0.0016 y^{5} \\
G(y)=1-1.3029 y+0.7809 y^{2}-1.0195 y^{3}+0.8809 y^{4}-0.4734 y^{5}
\end{gathered}
$$

In order to validate the present solution of the problem and find the accuracy, we will compare our solution and numerical results. Numerical solution of the problem is done with Maple package. The available methods in this software are a combination of the base scheme (midpoint or trapezoid), and a method enhancement scheme (deferred corrections or Richardson extrapolation). The numerical solution method is capable of handling both linear and nonlinear BVPs with fixed, periodic, and even nonlinear boundary conditions. A good agreement between the results of present technique and numerical solution is observed in Table 2, which confirms the validity of the proposed method. As it can be seen, error of the method is about in order of $1 \mathrm{e}-4$ to $1 \mathrm{e}-3$.

\section{RESULTS AND DISCUSSION}

Laminar flow and mass transfer of viscous fluid through a porous channel with moving or stationary wall in the presence of the chemical reaction is considered. The solution is obtained for velocity and mass concentration distributions versus governing parameter such as Reynolds number, Darcy number and suction/injection parameter. The results for this simulation were obtained for the case where $\varepsilon=0.7, k_{1}=0.1$ and $g=1$.

Fig. 2 shows the axial and normal velocity distribution and concentration filed for different values of Reynolds number. The value of parameters used in this simulating are: $\lambda=0, \gamma=0, \alpha=-1, \beta=0, D a=0.5, m=1$, $S c=0.65, X=2$. The result shows that the maximum value of the axial velocity of the fluid is increased by increasing the Reynolds number due to the acceleration of the fluid in the channel. Also, increasing the value of Reynolds number because increasing the normal velocity profile. Moreover, it can be seen that an increase in Re leads to an increase in the dimensionless concentration distribution and the maximum is shifted to the lower wall. Fig. 3 shows the axial velocity profile in both $X$ and $Y$ directions in the case of $\lambda=0, \gamma=-2, \alpha=-2, \beta=0$, $\operatorname{Re}=1, D a=0.5, m=1$ and $S c=0.65$. It is seen that by increasing parameter $X$, the amount of the maximum velocity of the fluid increases and it occurs in the same position of the wall according to $Y$ direction. 
Journal of Thermal Engineering, Research Article, Vol. 6, No. 2, Special Issue 11, pp. 24-40, March, 2020

Table 2. Comparison of the present results and numerical solution for $\lambda=0, \gamma=0, \alpha=-1, \beta=0$, $\operatorname{Re}=1, D a=0.5, \varepsilon=0.1, m=1, S c=0.65, g=1, k_{1}=0.1$.

\begin{tabular}{|l|l|l|l|l|l|l|l|}
\hline$y$ & \multicolumn{3}{|c|}{$F(y)$} & \multicolumn{3}{c|}{$G(y)$} \\
\hline & $\begin{array}{l}\text { Present } \\
\text { (Eq. 0) }\end{array}$ & $\begin{array}{l}\text { Numerical } \\
\text { solution }\end{array}$ & Error & $\begin{array}{l}\text { Present } \\
\text { Eq. 0) }\end{array}$ & $\begin{array}{l}\text { Numerical } \\
\text { solution }\end{array}$ & Error \\
\hline $\mathbf{0}$ & 1.0 & 1.0 & 0.0 & 1.0 & 1.0 & 0.0 \\
\hline $\mathbf{0 . 1}$ & 0.96991 & 0.96959 & $3.25 \mathrm{E}-4$ & 0.87658 & 0.87726 & 0.00068 \\
\hline $\mathbf{0 . 2}$ & 0.88979 & 0.88869 & $1.10 \mathrm{E}-3$ & & 0.76376 & 0.76505 & 0.00012 \\
\hline $\mathbf{0 . 3}$ & 0.77392 & 0.77186 & $2.06 \mathrm{E}-3$ & & 0.65797 & 0.65979 & 0.00018 \\
\hline $\mathbf{0 . 4}$ & 0.63563 & 0.63264 & $2.98 \mathrm{E}-3$ & & 0.55685 & 0.55911 & 0.00022 \\
\hline $\mathbf{0 . 5}$ & 0.48748 & 0.48382 & $3.65 \mathrm{E}-3$ & & 0.45889 & 0.46151 & 0.00026 \\
\hline $\mathbf{0 . 6}$ & 0.34136 & 0.33753 & $3.83 \mathrm{E}-3$ & & 0.36323 & 0.36615 & 0.00029 \\
\hline $\mathbf{0 . 7}$ & 0.20859 & 0.20525 & $3.34 \mathrm{E}-3$ & & 0.26943 & 0.27254 & 0.00031 \\
\hline $\mathbf{0 . 8}$ & 0.09991 & 0.09798 & $1.93 \mathrm{E}-3$ & & 0.17737 & 0.18045 & 0.00030 \\
\hline $\mathbf{0 . 9}$ & 0.02555 & 0.02619 & $6.35 \mathrm{E}-4$ & 0.08728 & 0.08969 & 0.00024 \\
\hline $\mathbf{1}$ & 0.0 & 0.0 & 0.0 & 0.0 & 0.0 & 0.0 \\
\hline
\end{tabular}

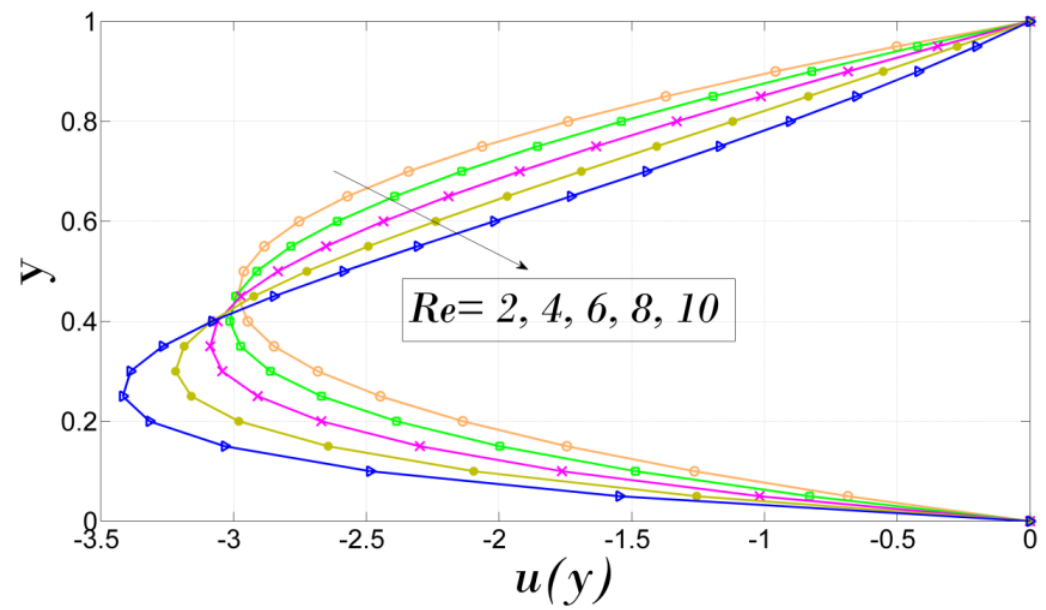

Figure 2. Effect of Re number on the velocity profiles and concentration distribution for $\lambda=0, \gamma=0, \alpha=-1, \beta=0, D a=0.5, m=1, S c=0.65, X=2$.

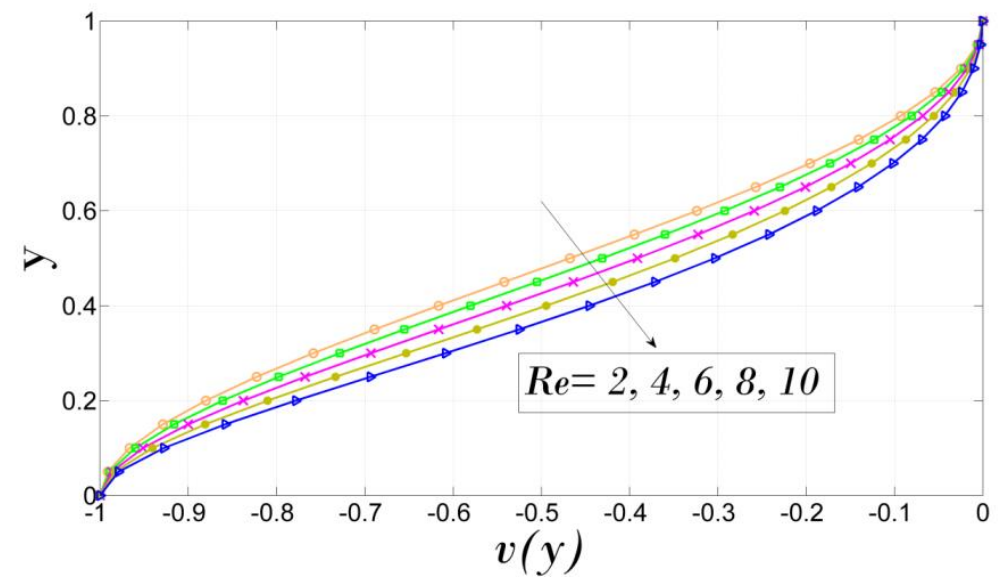


Journal of Thermal Engineering, Research Article, Vol. 6, No. 2, Special Issue 11, pp. 24-40, March, 2020
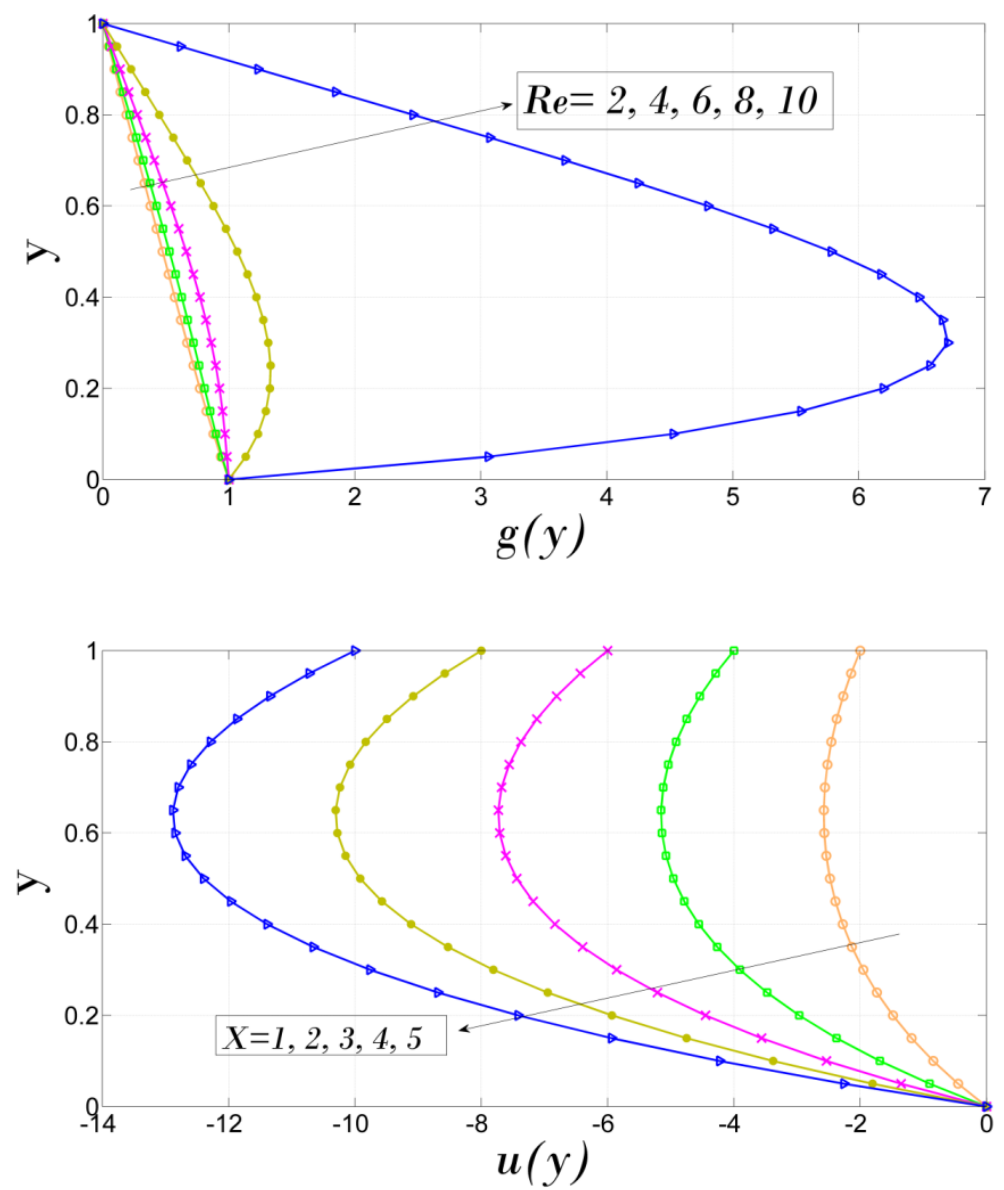

Figure 3. The effect of the parameter $\mathrm{X}$ on the axial velocity for $\lambda=0, \gamma=-2, \alpha=-2, \beta=0$,

$$
\operatorname{Re}=1, D a=0.5, m=1, S c=0.65 .
$$

Fig. 4 demonstrates how the axial and normal velocity profiles vary with the Darcy number. It is observed that by decreasing the Darcy number, the axial velocity profile becomes flatter due to high resistance of fluid flow when covering by the porous material with low permeability. Also, it can be seen that the normal velocity profile varies a little by varying the Darcy number.

Fig. 5 is plotted to show the effect of the axial velocity coefficient for bottom wall on axial and normal velocity profiles. Here the axial velocity initially increases by increasing $\lambda$ and then decreases after $y=0.25$. The normal velocity is decreased by increasing the value of $\lambda$.

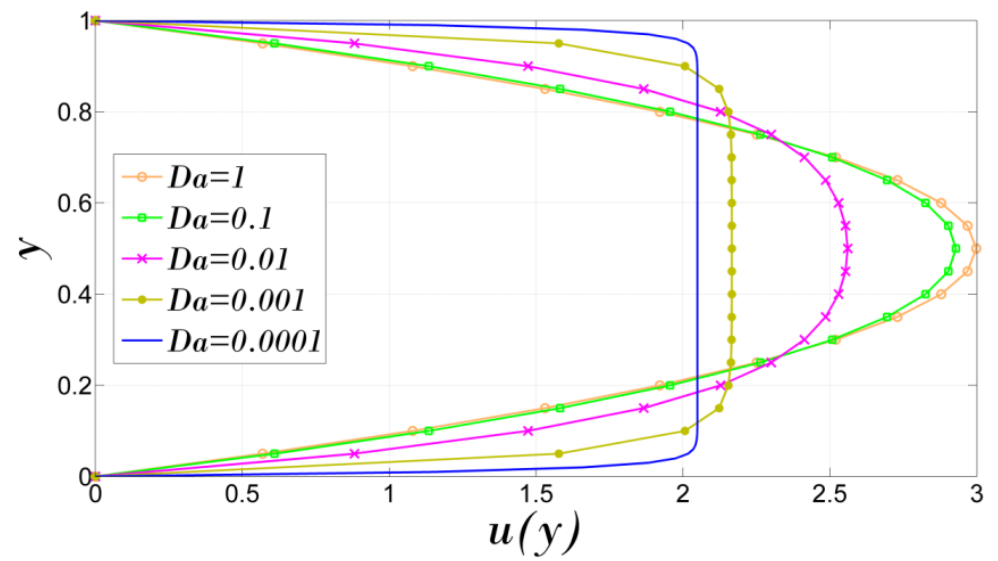


Journal of Thermal Engineering, Research Article, Vol. 6, No. 2, Special Issue 11, pp. 24-40, March, 2020

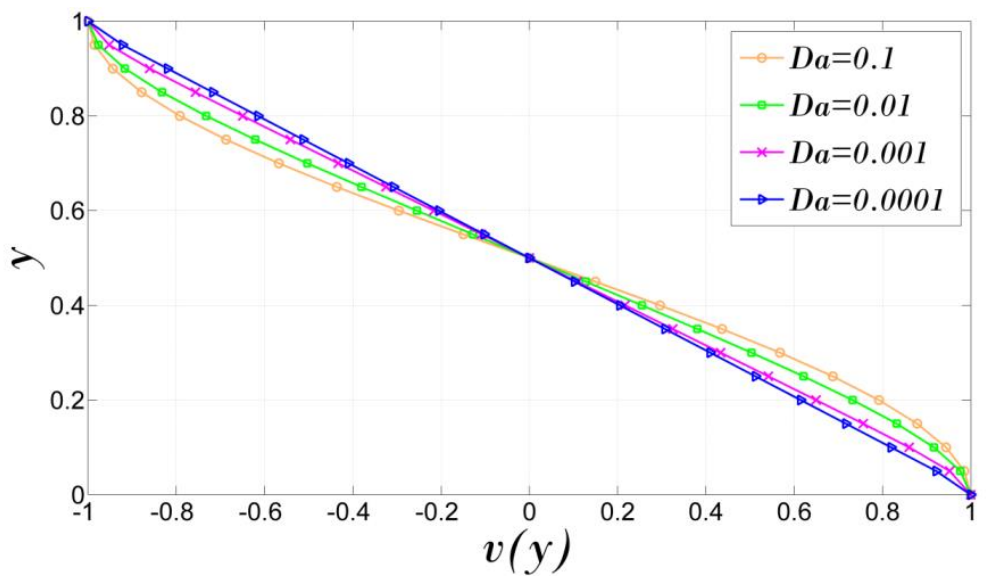

Figure 4. The velocity profiles for different Darcy numbers when $\lambda=0, \gamma=0, \alpha=1, \beta=-1$, $\operatorname{Re}=1, m=1, S c=0.65, X=1$.
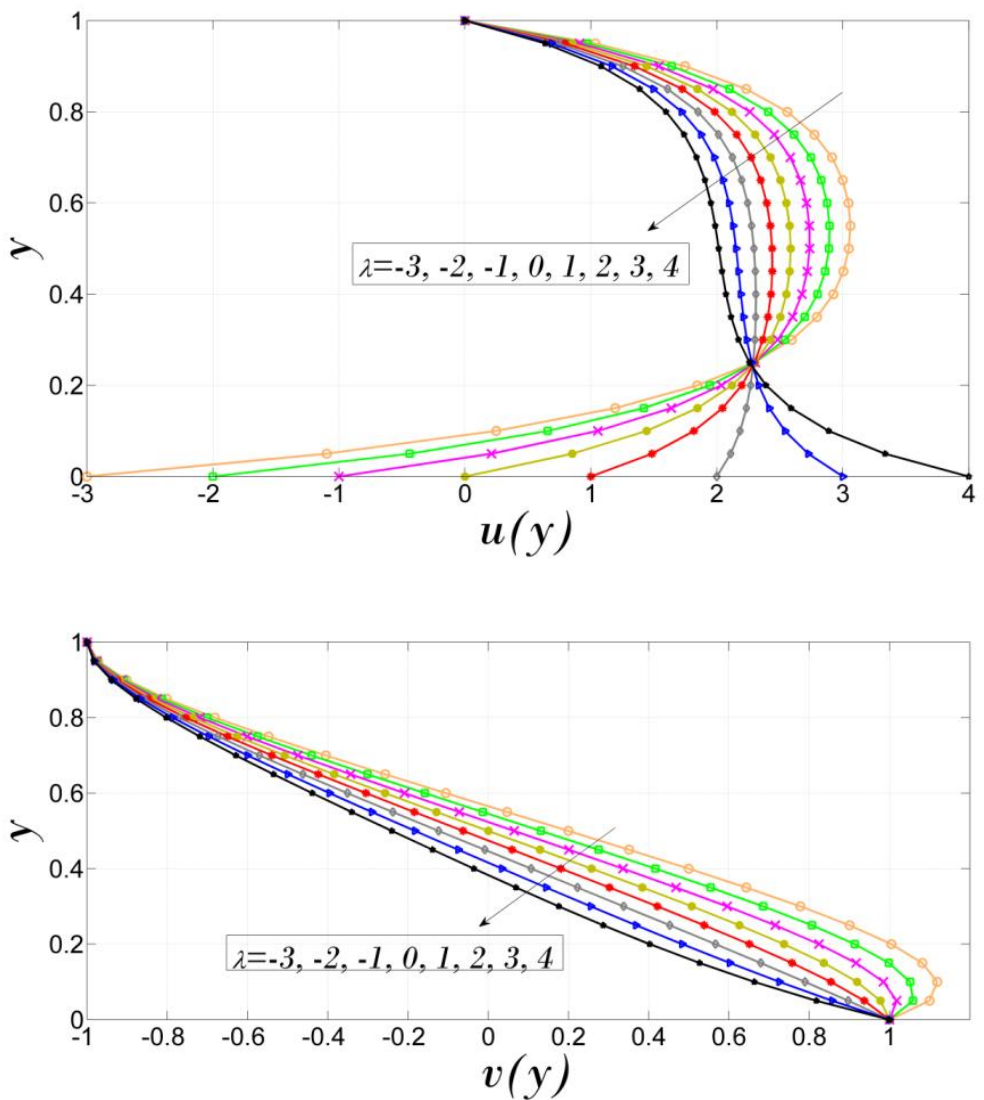

Figure 5. The effect of axial velocity coefficient for bottom wall $(\lambda)$ on velocity profiles when $\gamma=0, \alpha=1, \beta=-1, \operatorname{Re}=3, D a=0.01, m=0.5, S c=0.65, X=1$.

Fig. 6 shows the effect of suction/injection parameter from lower wall $(\alpha)$ on the velocity profiles for the case of moving walls. It is observed that for the case of $\alpha>0$ the maximum axial velocity of the fluid trends to the upper wall and for the case of $\alpha<0$ the minimum axial velocity of the fluid trends to the lower wall. Also it is shown 
Journal of Thermal Engineering, Research Article, Vol. 6, No. 2, Special Issue 11, pp. 24-40, March, 2020

that both the axial and normal velocity of the fluid is increased by increasing the suction/injection parameter so the fluid can penetrate faster through the porous medium.
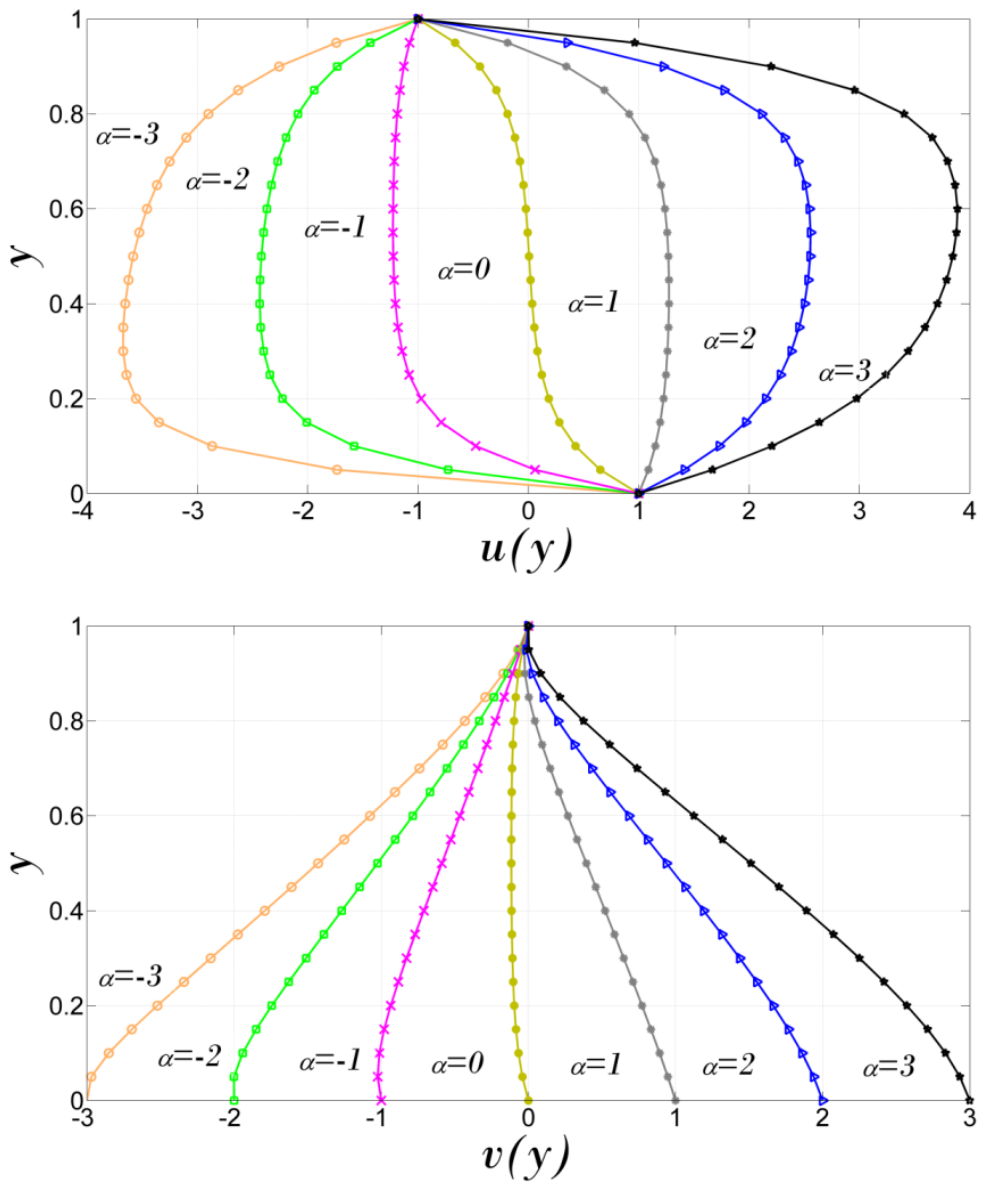

Figure 6. The effect of suction/injection parameter for bottom wall ( $\alpha$ ) on concentration distribution when

$$
\lambda=1, \gamma=-1, \beta=0, \operatorname{Re}=5, D a=0.01, m=0.5, S c=0.65, X=1 \text {. }
$$

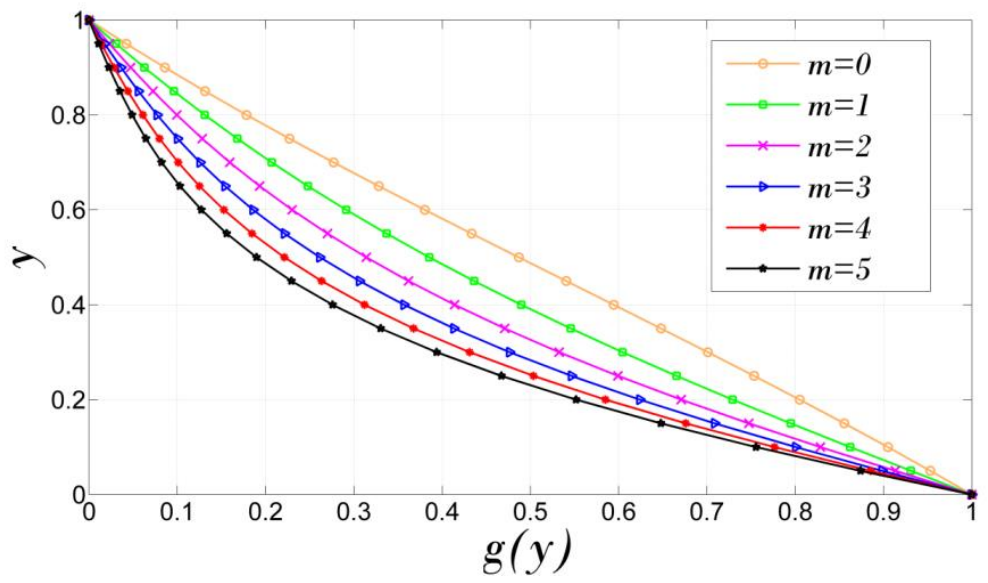

Figure 7. The effect of power law index of concentration model $(m)$ on velocity profiles when

$$
\lambda=1, \gamma=-1, \alpha=2, \beta=-1, \operatorname{Re}=1, D a=0.01, S c=0.65 \text {. }
$$

The influence of power-law index parameter $m$ on the concentration distribution for the case of moving walls is presented in Fig. 7. It is shown that increasing the power-law index parameter cause lower concentration of fluid. 
Journal of Thermal Engineering, Research Article, Vol. 6, No. 2, Special Issue 11, pp. 24-40, March, 2020

Fig. 8 presents the concentration distribution for various types of material with different Schmidt numbers at $\lambda=-1$ , $\gamma=1, \alpha=-1, \beta=-1, \operatorname{Re}=10, D a=0.1, m=5$. Schmidt number is defined as the ratio of momentum diffusivity (viscosity) and mass diffusivity and is used to characterize fluid flows in which there are simultaneous momentum and mass diffusion convection processes. It is seen that by decreasing the mass diffusivity, the concentration rate decreased due to the higher mass transfer rate.

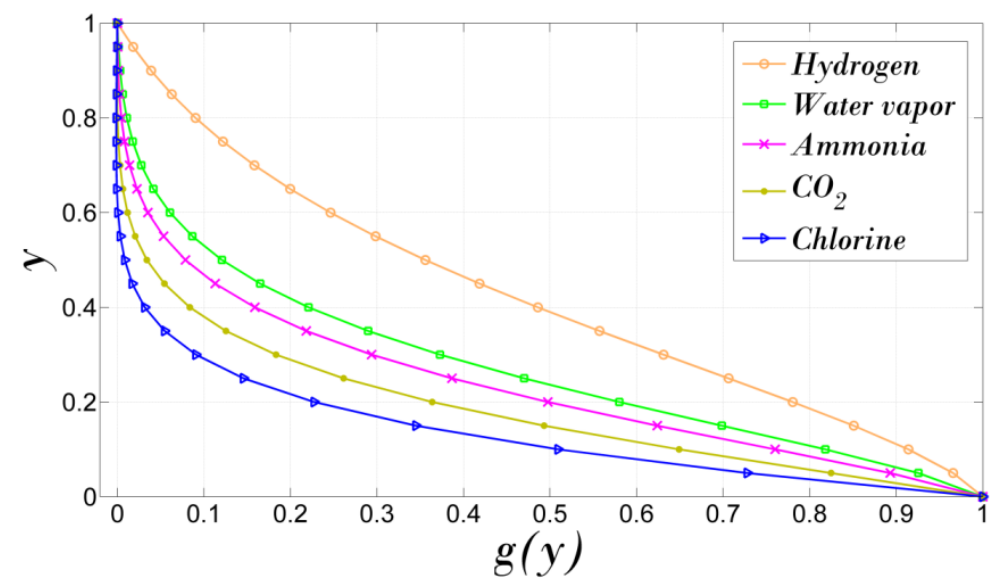

Figure 8. Concentration distribution for different types of materials when $\lambda=-1, \gamma=1, \alpha=-1$,

$$
\beta=-1, \operatorname{Re}=10, D a=0.1, m=5 .
$$

Table 3. Sherwood number for bottom wall of channel $S h_{B}$ when $\lambda=-1, \gamma=1, \alpha=0, \beta=0$

$D a=0.05$ and $m=5$.

\begin{tabular}{|l|l|l|l|l|l|}
\hline $\mathbf{R e}$ & \multicolumn{5}{|c|}{$S h_{B}$} \\
\hline & Hydrogen & Water vapor & Ammonia & $\mathrm{CO}_{2}$ & Chlorine \\
\hline $\mathbf{1}$ & 0.91708 & 0.75014 & 0.69843 & 0.60965 & 0.49437 \\
\hline $\mathbf{2}$ & 0.74681 & 0.22503 & 0.05984 & -0.22815 & -0.61078 \\
\hline $\mathbf{3}$ & 0.57198 & -0.3424 & -0.64183 & -1.17629 & -1.91319 \\
\hline $\mathbf{4}$ & 0.39227 & -0.95929 & -1.41952 & -2.26543 & -3.48875 \\
\hline $\mathbf{5}$ & 0.20735 & -1.63438 & -2.29006 & -3.53886 & -5.45910 \\
\hline $\mathbf{6}$ & 0.01684 & -2.37875 & -3.27576 & -5.06015 & -8.03674 \\
\hline $\mathbf{7}$ & -0.17963 & -3.20651 & -4.40689 & -6.92899 & -11.6168 \\
\hline $\mathbf{8}$ & -0.38259 & -4.13601 & -5.72564 & -9.30835 & -17.0265 \\
\hline $\mathbf{9}$ & -0.59245 & -5.19153 & -7.30167 & -12.4652 & -26.3929 \\
\hline $\mathbf{1 0}$ & -0.80976 & -6.41122 & -9.21538 & -16.9236 & -47.2205 \\
\hline
\end{tabular}

Table 3 presents the values of mass transfer in terms of Sherwood number for lower wall of the channel $\left(S h_{b}\right)$ for different values of Schmidt number and Reynolds number. It is seen that Sherwood number at the wall $Y=0$ decreases by increasing Schmidt number. Also, for all types of material (Hydrogen, water vapor, Ammonia, $\mathrm{CO}_{2}$ and Chlorine) Sherwood number is decreased by increasing Reynolds number. Furthermore, Sherwood number for the top wall is presented in Table 4. It is observed that the mass transfer rate at the wall $Y=1$ is decreased by increasing Schmidt number but the opposite effect is observed for the case of Reynolds number respect to the Sherwood number of bottom wall. 
Journal of Thermal Engineering, Research Article, Vol. 6, No. 2, Special Issue 11, pp. 24-40, March, 2020

Table 4. Sherwood number for top wall of channel $S h_{T}$ when $\lambda=-1, \gamma=1, \alpha=0, \beta=0$

$$
D a=0.05 \text { and } m=5 \text {. }
$$

\begin{tabular}{|l|l|l|l|l|l|}
\hline Re & \multicolumn{5}{|c|}{$S h_{T}$} \\
\hline & Hydrogen & Water vapor & Ammonia & $C_{2}$ & Chlorine \\
\hline $\mathbf{1}$ & 0.96836 & 0.91202 & 0.89628 & 0.87087 & 0.84060 \\
\hline $\mathbf{2}$ & 0.98375 & 0.96077 & 0.95589 & 0.94964 & 0.94516 \\
\hline $\mathbf{3}$ & 0.99965 & 1.01547 & 1.02441 & 1.04396 & 1.07711 \\
\hline $\mathbf{4}$ & 1.01608 & 1.07705 & 1.10357 & 1.15796 & 1.24677 \\
\hline $\mathbf{5}$ & 1.03309 & 1.14667 & 1.19563 & 1.29756 & 1.47080 \\
\hline $\mathbf{6}$ & 1.05071 & 1.22581 & 1.30362 & 1.47149 & 1.77906 \\
\hline $\mathbf{7}$ & 1.06901 & 1.31638 & 1.43169 & 1.69387 & 2.22667 \\
\hline $\mathbf{8}$ & 1.08803 & 1.42087 & 1.58566 & 1.98789 & 2.92812 \\
\hline $\mathbf{9}$ & 1.10783 & 1.54262 & 1.77610 & 2.39009 & 4.18019 \\
\hline $\mathbf{1 0}$ & 1.12848 & 1.68743 & 2.01307 & 2.97434 & 7.02889 \\
\hline
\end{tabular}

\section{CONCLUSION}

In this paper an analytical approach called Differential transformation method (DTM) has been applied to solve the problem of laminar flow and mass transfer of viscous fluid in a porous channel in the presence of chemical reaction with the moving or stationary walls. As a main outcome from the present study, the results are in excellent agreement with numerical ones. This method is accurate, efficient and powerful technique for solving the coupled nonlinear differential problems. Also, the effects of different parameters on velocity profile, concentration distribution and Sherwood number distribution have been presented. According to the results, increasing the Reynolds number causes to increase the fluid concentration. It is observed for the case of by decreasing the mass diffusivity (increasing Schmidt number), the fluid concentration is decreased due to higher mass transfer rate.

\section{NOMENCLATURE}

$C$

$C_{L}$

$C_{H}$

$D$

$D a$

De

DTM

H

$g$

G

K

$k_{1}$

$\operatorname{Re}$

Sc

$u, v$

$u_{L}, u_{H}$

$v_{L}, v_{H}$

$V$

$x$
Concentration of species of fluid

Concentration of lower wall of channel

Concentration of higher wall of channel

Diffusion coefficient of the diffusing spices

Darcy number

Deborah number

Differential transformation method

Channel width

Chemical reaction parameter

Dimensionless concentration function

Permeability of the porous medium

Reaction rate constant

Reynlods number

Schmidt number

Velocity component along $\mathrm{x}$ and $\mathrm{y}$ directions

Axial velocity at the lower and upper wall

Normal velocity at the lower and upper wall

Fluid velocity along y-direction

Coordinate along the channel 
Journal of Thermal Engineering, Research Article, Vol. 6, No. 2, Special Issue 11, pp. 24-40, March, 2020

$\begin{array}{ll}y & \text { Coordinate perpendicular to the channel } \\ \text { Greek symbols } & \text { Porosity of the porous medium } \\ \varepsilon & \text { Density of the fluid } \\ \mu & \text { Dynamic viscosity of the fluid } \\ \nu & \text { Kinetic viscosity of the fluid } \\ \alpha, \beta & \text { Suction/ injection parameter for lower and higher walls } \\ \lambda, \gamma & \text { Axial velocity coefficient of lower and higherwalls of the channel }\end{array}$

\section{REFERENCES}

[1] Cox SM. Two-dimensional flow of a viscous fluid in a channel with porous walls. Journal of Fluid Mechanics. 1991 Jun;227:1-33. doi:10.1017/S0022112091000010.

[2] Debruge LL, Han LS. Heat transfer in a channel with a porous wall for turbine cooling application. Journal of Heat Transfer, 1972, 94: 385-390. doi: 10.1115/1.3449956.

[3] Durst F, Haas R, Interthal W. The nature of flows through porous media. Journal of Non-Newtonian Fluid Mechanics. 1987 Jan 1;22(2):169-89. doi: 10.1016/0377-0257(87)80034-4.

[4] Rajagopal KR, Saccomandi G, Vergori L. Linear stability of Hagen-Poiseuille flow in a chemically reacting fluid. Computers \& Mathematics with Applications. 2011 Jan 1;61(2):460-9. doi: 10.1016/j.camwa.2010.11.026.

[5] Bég OA, Makinde OD. Viscoelastic flow and species transfer in a Darcian high-permeability channel. Journal of Petroleum Science and Engineering. 2011 Mar 1;76(3-4):93-9. doi: 10.1016/j.petrol.2011.01.008.

[6] Hayat T, Abbas Z. Channel flow of a Maxwell fluid with chemical reaction. Zeitschrift für angewandte Mathematik und Physik. 2008 Jan 1;59(1):124-44. doi: 10.1007/s00033-007-6067-1.

[7] Rundora L, Makinde OD. Effects of suction/injection on unsteady reactive variable viscosity non-Newtonian fluid flow in a channel filled with porous medium and convective boundary conditions. Journal of Petroleum Science and Engineering. 2013 Aug 1;108:328-35. doi: 10.1016/j.petrol.2013.05.010.

[8] Chinyoka T, Makinde OD. Computational dynamics of unsteady flow of a variable viscosity reactive fluid in a porous pipe. Mechanics Research Communications. 2010 Apr 1;37(3):347-53. doi: 10.1016/j.mechrescom.2010.02.007.

[9] Srinivas S, Muthuraj R. Effects of chemical reaction and space porosity on MHD mixed convective flow in a vertical asymmetric channel with peristalsis. Mathematical and Computer Modelling. 2011 Sep 1;54(56):1213-27. doi: 10.1016/j.mcm.2011.03.032.

[10] Makinde OD. Computer extension and bifurcation study by analytic continuation of porous tube flow. Jour. Math. Phys. Sci. 1996;30:1-24.

[11] Makinde OD. Extending the utility of perturbation series in problems of laminar flow in a porous pipe and a diverging channel. The ANZIAM Journal. 1999 Jul;41(1):118-28. doi: 10.1017/S0334270000011073.

[12] Makinde OD and Tay G. Numerical computation of bifurcation for steady flow in a converging channel with accelerating surface. A. M. S. E. Modelling, Measurement \& Control B. 1999: 68 (1): 33-43.

[13] Makinde OD, Moitsheki RJ. On nonperturbative techniques for thermal radiation effect on natural convection past a vertical plate embedded in a saturated porous medium. Mathematical Problems in Engineering. 2008;2008.

[14] Chinyoka T, Makinde OD. Viscoelastic modeling of the diffusion of polymeric pollutants injected into a pipe flow. Acta Mechanica Sinica. 2013 Apr 1;29(2):166-78. doi: 10.1007/s10409-013-0016-3.

[15] Makinde OD, Chinyoka T. Numerical investigation of buoyancy effects on hydromagnetic unsteady flow through a porous channel with suction/injection. Journal of Mechanical Science and Technology. 2013 May 1;27(5):1557-68. doi: 10.1007/s12206-013-0221-9.

[16] Patil PM, Kulkarni PS. Effects of chemical reaction on free convective flow of a polar fluid through a porous medium in the presence of internal heat generation. International Journal of Thermal Sciences. 2008 Aug 
Journal of Thermal Engineering, Research Article, Vol. 6, No. 2, Special Issue 11, pp. 24-40, March, 2020

1;47(8):1043-54. doi: 10.1016/j.ijthermalsci.2007.07.013.

[17] Subramanyam Reddy A, Srinivas S, Ramamohan TR. Analysis of heat and chemical reaction on an asymmetric laminar flow between slowly expanding or contracting walls. Heat Transfer-Asian Research. 2013 Jul;42(5):422-43. doi: 10.1002/htj.21036.

[18] Matin MH, Pop I. Forced convection heat and mass transfer flow of a nanofluid through a porous channel with a first order chemical reaction on the wall. International Communications in Heat and Mass Transfer. 2013 Aug 1;46:134-41. doi: 10.1016/j.icheatmasstransfer.2013.05.001.

[19] Sivaraj R, Kumar BR. Chemically reacting dusty viscoelastic fluid flow in an irregular channel with convective boundary. Ain Shams Engineering Journal. 2013 Mar 1;4(1):93-101. doi: 10.1016/j.asej.2012.06.005.

[20] Srinivas S, Gupta A, Gulati S, Reddy AS. Flow and mass transfer effects on viscous fluid in a porous channel with moving/stationary walls in presence of chemical reaction. International communications in heat and mass transfer. 2013 Nov 1;48:34-9. doi: 10.1016/j.icheatmasstransfer.2013.09.002.

[21] Mahdy A. Effect of chemical reaction and heat generation or absorption on double-diffusive convection from a vertical truncated cone in porous media with variable viscosity. International Communications in Heat and Mass Transfer. 2010 May 1;37(5):548-54. doi: 10.1016/j.icheatmasstransfer.2010.01.007.

[22] Rashidi MM, Shahmohamadi H, Dinarvand S. Analytic Approximate Solutions for Unsteady TwoDimensional and Axisymmetric Squeezing Flows between Parallel Plates. Mathematical Problems in Engineering. 2008: Article ID 935095. doi: 10.1155/2008/935095.

[23] Dinarvand S, Rashidi M, Doosthoseini A. Analytical approximate solutions for two-dimensional viscous flow through expanding or contracting gaps with permeable walls. Open Physics. 2009 Dec 1;7(4):791-9. doi: 10.2478/s11534-009-0024-x.

[24] Rashidi MM, Domairry G, Dinarvand S. Approximate solutions for the Burger and regularized long wave equations by means of the homotopy analysis method. Communications in Nonlinear Science and Numerical Simulation. 2009 Mar 1;14(3):708-17. doi: 10.1016/j.cnsns.2007.09.015.

[25] Rashidi MM, Ganji DD, Dinarvand S. Approximate traveling wave solutions of coupled Whitham-Broer-Kaup shallow water equations by homotopy analysis method. International Journal of Differential Equations. 2008 May 15;2008: Article ID 243459. doi: 10.1155/2008/243459.

[26] Raftari B, Yildirim A. The application of homotopy perturbation method for MHD flows of UCM fluids above porous stretching sheets. Computers \& Mathematics with Applications. 2010 May 1;59(10):3328-37. doi: 10.1016/j.camwa.2010.03.018.

[27] Esmaeilpour M, Ganji DD. Application of He's homotopy perturbation method to boundary layer flow and convection heat transfer over a flat plate. Physics Letters A. 2007 Dec 10;372(1):33-8. doi: 10.1016/j.physleta.2007.07.002.

[28] Rashidi MM, Ganji DD, Dinarvand S. Explicit analytical solutions of the generalized Burger and BurgerFisher equations by homotopy perturbation method. Numerical Methods for Partial Differential Equations: An International Journal. 2009 Mar;25(2):409-17. doi: 10.1002/num.20350.

[29] Mohyud-Din ST, Yildirim A, Anıl Sezer S, Usman M. Modified variational iteration method for freeconvective boundary-layer equation using padé approximation. Mathematical Problems in Engineering. 2010;2010: Article ID 318298. doi: 10.1155/2010/318298.

[30] Rashidi MM, Shahmohamadi H. Analytical solution of three-dimensional Navier-Stokes equations for the flow near an infinite rotating disk. Communications in Nonlinear Science and Numerical Simulation. $2009 \mathrm{Jul}$ 1;14(7):2999-3006. doi: 10.1016/j.cnsns.2008.10.030.

[31] Wazwaz AM. The variational iteration method for solving two forms of Blasius equation on a half-infinite domain. Applied Mathematics and Computation. 2007 May 1;188(1):485-91. doi: 10.1016/j.amc.2006.10.009.

[32] Wazwaz AM. The modified decomposition method and Padé approximants for a boundary layer equation in unbounded domain. Applied Mathematics and Computation. 2006 Jun 15;177(2):737-44. doi: 10.1016/j.amc.2005.09.102.

[33] Alizadeh E, Sedighi K, Farhadi M, Ebrahimi-Kebria HR. Analytical approximate solution of the cooling problem by Adomian decomposition method. Communications in Nonlinear Science and Numerical 
Journal of Thermal Engineering, Research Article, Vol. 6, No. 2, Special Issue 11, pp. 24-40, March, 2020

Simulation. 2009 Feb 1;14(2):462-72. doi: 10.1016/j.cnsns.2007.09.008.

[34] Kechil SA, Hashim I. Non-perturbative solution of free-convective boundary-layer equation by Adomian decomposition method. Physics Letters A. 2007 Mar 19;363(1-2):110-4. doi: 10.1016/j.physleta.2006.11.054

[35] Zhou, J. Differential transformation and its applications for electrical circuits. ed: Huazhong University Press. 1986. Wuhan. China.

[36] Ayaz F. Applications of differential transform method to differential-algebraic equations. Applied Mathematics and Computation. 2004 May 13;152(3):649-57. doi: 10.1016/S0096-3003(03)00581-2.

[37] Liu H, Song Y. Differential transform method applied to high index differential-algebraic equations. Applied Mathematics and Computation. 2007 Jan 15;184(2):748-53. doi: 10.1016/j.amc.2006.05.173.

[38] Mosayebidorcheh S, Sheikholeslami M, Hatami M, Ganji DD. Analysis of turbulent MHD Couette nanofluid flow and heat transfer using hybrid DTM-FDM. Particuology. 2016 Jun 1;26:95-101. doi: 10.1016/j.partic.2016.01.002.

[39] Mosayebidorcheh S, Hatami M, Ganji DD, Mosayebidorcheh T, Mirmohammadsadeghi SM. Investigation of Transient MHD Couette flow and Heat Transfer of Dusty Fluid with Temperature-Dependent Oroperties. Journal of Applied Fluid Mechanics. 2015 Sep 1;8(4).

[40] Hatami M, Ghasemi SE, Sahebi SA, Mosayebidorcheh S, Ganji DD, Hatami J. Investigation of third-grade non-Newtonian blood flow in arteries under periodic body acceleration using multi-step differential transformation method. Applied Mathematics and Mechanics. 2015 Nov 1;36(11):1449-58. doi: 10.1007/s10483-015-1995-7.

[41] Mosayebidorcheh S, Rahimi-Gorji M, Ganji DD, Moayebidorcheh T, Pourmehran O, Biglarian M. Transient thermal behavior of radial fins of rectangular, triangular and hyperbolic profiles with temperature-dependent properties using DTM-FDM. Journal of Central South University. 2017 Mar 1;24(3):675-82. doi: 10.1007/s11771-017-3468-y.

[42] Mosayebidorcheh S, Rahimi-Gorji M, Ganji DD, Moayebidorcheh T, Pourmehran O, Biglarian M. Transient thermal behavior of radial fins of rectangular, triangular and hyperbolic profiles with temperature-dependent properties using DTM-FDM. Journal of Central South University. 2017 Mar 1;24(3):675-82. doi: 10.1007/s11771-017-3468-y.

[43] Mosayebidorcheh S, Farzinpoor M, Ganji DD. Transient thermal analysis of longitudinal fins with internal heat generation considering temperature-dependent properties and different fin profiles. Energy conversion and management. 2014 Oct 1;86:365-70. doi: 10.1016/j.enconman.2014.05.033.

[44] Odibat ZM. Differential transform method for solving Volterra integral equation with separable kernels. Mathematical and Computer Modelling. 2008 Oct 1;48(7-8):1144-9. doi: 10.1016/j.mcm.2007.12.022.

[45] Arikoglu A, Ozkol I. Solutions of integral and integro-differential equation systems by using differential transform method. Computers \& Mathematics with Applications. 2008 Nov 1;56(9):2411-7. doi: 10.1016/j.camwa.2008.05.017.

[46] Arikoglu A, Ozkol I. Solution of boundary value problems for integro-differential equations by using differential transform method. Applied Mathematics and Computation. 2005 Sep 15;168(2):1145-58. doi: 10.1016/j.amc.2004.10.009.

[47] Mosayebidorcheh S. Solution of the boundary layer equation of the power-law pseudoplastic fluid using differential transform method. Mathematical Problems in Engineering. 2013: Article ID 685454. doi: $10.1155 / 2013 / 685454$.

[48] Mosayebidorcheh S, Mosayebidorcheh T. Series solution of convective radiative conduction equation of the nonlinear fin with temperature dependent thermal conductivity. International Journal of Heat and Mass Transfer. 2012 Nov 1;55(23-24):6589-94. doi: 10.1016/j.ijheatmasstransfer.2012.06.066.

[49] Mosayebidorcheh S. Taylor series solution of the electrohydrodynamic flow equation. Journal of Mechanical Engineering and Technology. 2013 Sep;1(2):40-5.

[50] Mosayebidorcheh S. Analytical investigation of the micropolar flow through a porous channel with changing walls. Journal of Molecular Liquids. 2014 Aug 1;196:113-9. doi: 10.1016/j.molliq.2014.03.022.

[51] Mosayebidorcheh S. Comments on "Numerical solutions of the space-and time-fractional coupled Burgers 
Journal of Thermal Engineering, Research Article, Vol. 6, No. 2, Special Issue 11, pp. 24-40, March, 2020

equations by generalized differential transform method, Applied Mathematics and Computation 217 (2011) 7001-7008". Applied Mathematics and Computation. 2014 Sep 15;243:960-2. doi: 10.1016/j.amc.2014.06.055.

[52] Odibat Z, Momani S, Erturk VS. Generalized differential transform method: application to differential equations of fractional order. Applied Mathematics and Computation. 2008 Apr 1;197(2):467-77. doi: 10.1016/j.amc.2007.07.068.

[53] Erturk VS, Momani S, Odibat Z. Application of generalized differential transform method to multi-order fractional differential equations. Communications in Nonlinear Science and Numerical Simulation. 2008 Oct 1;13(8):1642-54. doi: 10.1016/j.cnsns.2007.02.006.

[54] Arikoglu A, Ozkol I. Solution of fractional differential equations by using differential transform method. Chaos, Solitons \& Fractals. 2007 Dec 1;34(5):1473-81. doi: 10.1016/j.chaos.2006.09.004.

[55] Reza Seyf H, Moein Rassoulinejad-Mousavi S. An analytical study for fluid flow in porous media imbedded inside a channel with moving or stationary walls subjected to injection/suction. Journal of fluids engineering. 2011 Sep 1;133(9). doi: 10.1115/1.4004822. 